around airports between aircraft nominally separated by given amounts, laterally, vertically and longitudinally? If this were done, nominal separation standards could be specified for each area to give a specified safety standard; they might well be found to be quite different from those currently used. Such calculations might show whether the collision risk is less on airways or off airways. Publication of traffic density maps might help pilots avoid volumes of highest traffic density.

The magnitude of altitude error makes desirable height control for all automatic pilots, the standardization of altimeters and the investigation whether some other variable than pressure might more accurately be used to provide vertical separation, particularly at great heights.

\title{
Visual Awareness and Instrument Design
}

\author{
from W. H. McKinlay \\ (Smiths Aircraft lnstruments Ltd.)
}

THOSE of us who are concerned with the development of flight instruments feel that the automatic pilot can be used to control the aircraft throughout the traffic pattern, and in fact, the major British system at present in service is particularly designed to provide this facility. The fact that the pilot is then free to concentrate more on events outside the cockpit has incidental advantages from our point of view, as the resulting visual reference provides by far the best method of detecting an automatic pilot malfunction should it occur in conditions of good visibility.

Although we do not at present provide full automatic landing, it is worth pointing out that we do provide control to the fine limits required for such an operation. Its introduction awaits a number of other factors, notably the provision of a radio guidance system superior to ILS during the critical final phase of the approach. We will also have to pay particular attention to safety. No electrical circuit is of itself absolutely infallible and we will have to consider what degree of multiplicity is necessary to achieve the required safety under realistic fault conditions.

The philosophy of design for safety will involve the complete control loop including, of course, any ground installation required, and this may call for international agreement. The safety aspect of the problem appears whenever electrical techniques are used in a role which directly affects the safety of an aircraft. If the collision problem is eventually solved electrically, the standard of overall system safety required will become more exacting the more aircraft operators have to depend on the equipment in congested air spaces. In the guidance and control field it is our experience that it is vital to work to a design philosophy which recognizes that equipment failures will sometimes occur, and which provides for the inclusion of failure warnings with duplication or even triplication where necessary. All this may sound pessimistic, but no amount of engineering detail can produce a higher degree of safety than is permitted by the basic conception of a system. Perhaps the systems currently being proposed for collision warning should be subject to this sort of analysis once they have survived the initial one of practical feasibility. 


\section{from Captain D. Martin}

THE immediate solutions to the problem of collision seem to be: (I) airborne radar or some self-contained device; (2) better ground control; (3) increased vigilance on the part of the pilots. As most accidents seem to occur in aerodomecontrolled areas in good visibility, the third possibility appears to be the simplest and the most obvious for an immediate lowering of the accident rate. It is clear that pilots are not exercising vigilance in control zones and the reason in my opinion is that it is a physical impossibility in modern airliners to look out of the cockpit for more than brief periods because of the heavy workloads imposed by these machines. Since the end of the war, aircraft have become more complicated and faster and crews have been reduced. Thus at the end of what is frequently an exhausting trip the pilot has to penetrate into dense traffic zones where extreme alertness is required.

It has been said that pilots should not look at instruments in control zones in good weather. Whilst very sound in theory, this is not always possible. For example, very complicated patterns are marked in colours on the approach chart, but these colours are not marked on the ground, consequently the pilot must use his instruments even in good visibility. During the time that the captain is thus occupied, the first officer has to tune in to anything up to six beacons, and has 20 or more items to check on his list, all in the space of 4 to Io minutes. In addition both pilots will have to listen to anything up to 20 instructions from the ground with 3 or more changes of frequency. Very often it happens that the only time there is to keep a sharp lookout is in the last few seconds on final approach when everything has been done and there is nothing to do but to land the aircraft.

For this aspect of the flight there would seem, therefore, to be only two possible solutions: (1) a reduction of work load in the cockpit; (2) better ground control.

\section{NAVIGATION AND AIR TRAFFIC CONTROL}

THE Ausschuss für Funkortung is holding a meeting in Berlin from 19 to 24 May to hear papers on air navigation and air traffic control. Further details may be had on application to this Institute. 